\title{
ON SOME BANACH SPACE SEQUENCES
}

\author{
ROSHDI KHALIL
}

We introduce the Banach space of yector valued sequences $2^{p}, q(E), \quad 1 \leq p, q \leq \infty$, where $E$ is a Banach space. Then we study the relation between $\imath^{p, q}\langle E\rangle$ and the schur multipliers of $\imath^{P} \hat{\otimes} E$, where $E$ is taken to be some $\imath^{r}$.

\section{Introduction}

Let $E$ be a Banach space. Cohen [3], used the spaces $Z^{P}(E), Z^{P}\{E\}$ together with the space he introduced ${ }_{2} P_{\langle E\rangle}$, to study p-summing operators, and their dual ideal (see [11]). Apiola [1], studied the duality relationships between the spaces $\imath^{p}(E), \imath^{P}\{E\}$ and $\imath^{P}\langle E\rangle$.

In this paper we introduce the space $\imath^{p, q}\langle E\rangle$, and find its dual. Further, we investigate the relationship between such spaces and the Schur multipliers [2], on discrete spaces.

Throughout the paper, if $E$ and $F$ are Banach spaces, then $E \hat{\otimes} E$ and $E \check{\otimes} F$ will denote the completion of the projective tensor product of $E$ with $F$, and the injective tensor product, respectively [4]. Let $\phi \in E \otimes F ;$ then $\|\phi\|_{\pi}$ designates the projective norm and $\|\phi\|_{\varepsilon}$ that of the infective norm. The dual of $E$ will be denoted by $E^{*}$ for any Banach space $E$. The set of natural numbers is denoted by $N$, and the complex numbers by $\mathbb{C}$. Let $\tau^{p}$ be the space of $p$-summable sequences, $1 \leq p \leq \infty$. 


\section{The space $\imath^{p, q}\langle E\rangle$ and its dual}

Let $E$ be a Banach space. Then $Z^{P}(E)$ will denote the space of all functions $f: N \rightarrow E$, such that $\sum_{n=1}^{\infty}\left|\left\langle f(n), x^{*}\right\rangle\right|^{p}<\infty, x^{*} \in E^{*}$. The space $l^{P}(E)$ becomes a Banach space when one introduces the norm

$$
\|f\|_{\varepsilon(p)}=\sup _{x^{*}}\left\{\left(\sum\left|\left\langle f(i), x^{*}\right\rangle\right|^{p}\right)^{1 / p},\left\|x^{*}\right\| \leq 1, x^{*} \in E^{*}\right\},
$$

for all $f \in 2^{p}(E),[3]$. Grothendieck, [5], showed that $Z^{p}(E)$ is isometrically isomorphic to $\left(\imath^{p^{\prime}} \hat{\otimes} F\right)^{*}$, where $F^{*}=E$, and $1 / p+1 / p^{\prime}=1$.

Cohen, [3], introduced the space $l^{p}\langle E\rangle$ to be the space of all functions $f: N+E$ such that $\sum_{i=1}^{\infty}|\langle f(i), g(i)\rangle|<\infty$, for all $g \in z^{p^{\prime}}\left(E^{*}\right)$. The norm of $f$ is given by

$$
\|f\|_{\sigma(1, p)}=\sup _{g}\left\{\sum_{i=1}^{\infty}|(f(i), g(i))|, g \in \tau^{P^{\prime}}\left(E^{*}\right) \text { and }\|g\|_{\varepsilon\left(p^{\prime}\right)} \leq 1\right\} \text {. }
$$

The space $\imath^{p}(E)$ was shown to induce the injective norm on $\imath^{p} \otimes E,[3]$, and Cohen showed that $Z^{P}(E)$ induces the projective norm on $\imath^{P} \otimes E$. Further, Apiola, [1], showed that $\left(Z^{P}(E)\right) * \equiv Z^{P^{\prime}}\left(E^{*}\right)$ and $\left(\imath^{p}(E)\right) * \equiv \tau^{p}\left(E^{*}\right)$.

Now we introduce the space $\imath^{p, q}\langle E\rangle$ to be the space of all functions $f: N \rightarrow E$ such that $\sum_{i=1}^{\infty}|\langle f(i), g(i)\rangle|^{p}<\infty$ for all $g \in l^{q^{\prime}}\left(E^{*}\right)$. If $f \in \imath^{p, q}\langle E\rangle$, then we define

$$
\|f\|_{\sigma(p, q)}=\sup _{g}\left(\sum_{i=1}|\langle f(i), g(i)\rangle|^{p}\right)^{I / p},
$$

where $g \in \imath^{q^{\prime}}\left(E^{*}\right)$ and $\|g\|_{\varepsilon\left(q^{\prime}\right)} \leq 1$. 
LEMMA 1.1. The function \|\|$_{\sigma(p, q)}$ is a norm on $\imath^{p, q(E)}$.

Proof. It is enough to show that $\|f\|_{\sigma(p, q)}<\infty$ for all $f \in \tau^{p, q}(E)$. The rest of the properties of the norm are easy to verify.

Let $f \in \imath^{p, q}\langle E\rangle$. Define the bilinear form

$$
\begin{gathered}
\hat{f}: z^{p^{\prime}} \times \imath^{q^{\prime}}\left(E^{*}\right)-\mathbf{C}, \\
f(a, g)=\sum_{i=1}^{\infty} a(i)(f(i), g(i)) .
\end{gathered}
$$

It is not hard to check that $\hat{f}$ is separately continuous on $l^{p^{\prime}} \times \tau^{q^{\prime}}\left(E^{*}\right)$. Hence, [2, p. 172], $\hat{f}$ is jointly continuous, and consequently $\|f\|_{\sigma(p, q)}<\infty$ for all $f \in l^{p, q}(E)$.

THEOREM 1.2. The space $\tau^{p, q}\langle E\rangle$ with the $\sigma(p, q)$ norm is a Banach space.

Proof. Let $f_{n} \in z^{p, q}\langle E\rangle$ such that $\sum_{n=1}^{\infty}\left\|f_{n}\right\|_{\sigma(p, q)}<\infty$. It is enough to show that $\left\|\sum_{n=1}^{\infty} f_{n}\right\|<\infty,[12]$. We first prove this for the case $p=1$. Since $E$ is a Banach space, then every absolutely summable sequence in $E$ is summable. It follows that for each natural number $i$, the serries $\sum_{n=1}^{\infty} f_{n}(i)$ is convergent in $E$. Define $F: N \rightarrow E$ by $F(i)=\sum_{n=1}^{\infty} f_{n}(i)$.

Let $g \in \tau^{q^{\prime}}\left(E^{*}\right)$ and $\|g\|_{\varepsilon\left(q^{\prime}\right)} \leq 1$. We have to prove that $\sum_{i=1}^{\infty}|(E(i), g(i))|<\infty$. Now 
$\sum_{i=1}^{\infty}|\langle F(i), g(i)\rangle|$

$$
\begin{aligned}
& =\sum_{i=1}^{\infty}\left|\left\langle\sum_{n=1}^{\infty} f_{n}(i), g(i)\right\rangle\right| \\
& =\sum_{i=1}^{\infty}\left|\sum_{n=1}^{\infty}\left\langle f_{n}(i), g(i)\right\rangle\right| \quad\left(\text { since } \sum_{n=1}^{\infty}\left\|f_{n}(i)\right\|<\infty\right) \\
& \leq \sum_{i=1}^{\infty} \sum_{n=1}^{\infty}\left|\left\langle f_{n}(i), g(i)\right\rangle\right| \quad\left(\text { since }\left|\sum_{n=1}\left\langle f_{n}(i), g(i)\right\rangle\right|<\infty\right) .
\end{aligned}
$$

If $v$ is the counting measure on the set of natural numbers $N$, then

$$
\sum_{i=1}^{\infty} \sum_{n=1}^{\infty}\left|\left\langle f_{n}(i), g(i)\right\rangle\right|
$$

can be considered as

$$
\int_{N} \sum_{n=1}\left|\left\langle f_{n}(i), g(i)\right\rangle\right| d v(i)
$$

As a consequence of the monotone convergence theorem we get

$$
\int_{N} \sum_{n=1}^{\infty}\left|\left\langle f_{n}(i), g(i)\right\rangle\right| d \nu(i)=\sum_{n=1}^{\infty} \int_{N}\left|\left\langle f_{n}(i), g(i)\right)\right| d v(i) \text {. }
$$

It follows that

$$
\begin{aligned}
& \sum_{i=1}^{\infty}|\langle F(i), g(i)\rangle| \\
& \left.\quad \leq \sum_{i=1}^{\infty} \sum_{n=1}^{\infty}\left|\left(f_{n}(i), g(i)\right)\right|<\infty \quad \text { (since } \sum_{n=1}^{\infty}\left\|f_{n}\right\|_{\sigma(1, q)}<\infty\right) .
\end{aligned}
$$

Hence $\sum_{i=1}^{\infty}|\langle F(i), g(i)\rangle|<\infty$ for all $g \in z^{q^{\prime}}\left(E^{*}\right)$ with $\|g\|_{\varepsilon\left(q^{\prime}\right)}<\infty$. Consequently $F \in \imath^{1, q}\langle E\rangle$, and so $\sum_{n=1}^{\infty} f_{n} \in \imath^{1, q}\langle E\rangle$.

For general $p$, the result follows from the fact that

$$
\|f\|_{\sigma(p, q)}=\sup _{\theta, g}\left|\sum_{i=1}^{\infty} \theta(i)(f(i), g(i))\right|,
$$


where $\theta \in Z^{p^{\prime}}, g \in Z^{q^{\prime}}\left(E^{*}\right)$ and $\|\theta\|_{p^{\prime}} \leq 1,\|g\|_{\varepsilon\left(q^{\prime}\right)} \leq 1$. Hence the proof of the theorem is complete.

Let $Z^{r} \cdot l^{s}\left(E^{*}\right)$ be the set of all elements of the form $a \cdot f$ such that $a \in Z^{r}, f \in Z^{s}\left(E^{*}\right)$ and $(a \cdot f)(i)=a(i) \cdot f(i)$.

THEOREM 1.3. A Zinear functional $F$ on $\imath^{p, q}\langle E\rangle$ is bounded if and only if $F$ is of the form $a \cdot f$, for some $a \in \eta^{P^{\prime}}$ and $f \in \eta^{q^{\prime}}\left(E^{*}\right)$.

REMARK. The space $l^{l, q}(E)$ is just $l^{q}(E)$ in Cohen [3]. Apiola, [1], proved that $\left(l^{l}, q_{|E\rangle}\right) *$ is isometrically isomorphic to $l^{q^{\prime}}\left(E^{*}\right)$ which is in turn isomorphic to $\tau^{\infty} \cdot \tau^{q^{\prime}}\left(E^{*}\right)$.

Proof of Theorem 1.3. Let $a \in Z^{P^{\prime}}$ and $f \in Z^{q^{\prime}}\left(E^{*}\right)$. Consider the linear functional $F: \imath^{p, q}(E) \rightarrow \mathbb{C}$ defined by

$$
F(g)=\sum_{i=1}^{\infty} a(i)(f(i), g(i)) .
$$

Then

$$
|F(g)| \leq\|a\|_{p} \cdot\|f\|_{\varepsilon\left(q^{\prime}\right)^{\cdot}\|g\|_{\sigma(p, q)}}
$$

Hence $F$ is bounded and $\|F\| \leq\|a\|_{p^{\prime}} \cdot\|f\|_{\varepsilon\left(q^{\prime}\right)}$.

$$
\text { Conversely, let } F \in\left(\imath^{p, q(E)}\right) * \text {. Hence }|F(f)| \leq \lambda \cdot\|f\|_{\sigma(p, q)} \text { for }
$$

some constant $\lambda$. Let $e_{i}$ be the natural embedding of $E$ in $\imath^{p, q}\langle E\rangle$, so

$$
e_{i}(x)(j)= \begin{cases}x, & i=j, \\ 0, & i \neq j .\end{cases}
$$

Put $x_{i}^{*}=F \circ e_{i} \cdot$ Clearly $x_{i}^{*} \in E^{*}$, and if $f \in \imath^{p, q}\langle E\rangle$, then

$$
F(f)=\sum_{i=1}^{\infty}\left\langle f(i), x_{i}^{*}\right\rangle .
$$

Assume $F$ to be of norm one; then there is an $a \in 2^{p^{\prime}}$ such that 
$\|a\|_{p^{\prime}} \leq 1$ and $|F(f)| \leq \sup _{g} \sum_{i=1}^{\infty}|a(i)(f(i), g(i)\rangle|,\|g\|_{\varepsilon(q)} \leq 1$. Thus

$$
\left|\sum_{i=1}^{\infty}\left\langle f(i), x_{i}^{*}\right\rangle\right| \leq \sup _{g} \sum_{i=1}^{\infty} \mid a i\langle f(i), g(i)\rangle,\|g\|_{\varepsilon\left(q^{\prime}\right)} \leq 1 .
$$

Now let $D$ be the unit disc and $\pi D$ be the countable product of $D$ with itself. Since $D$ is compact, then $\pi D$ is compact. Let $B_{1}$ be the unit ball of $l^{q^{\prime}}\left(E^{*}\right)$. As a dual of $l^{l}, q_{\langle E\rangle},[1], B_{1}$ is compact with respect to the $w^{*}$-topology, and so is the product space $\pi D \times B_{1}$. Let $C\left(\pi D \times B_{1}\right)$ be the space of continuous functions on $\pi D \times B_{1}$. Consider the map

$$
\psi:{ }_{2}^{p}, q\langle E\rangle \rightarrow C\left(\pi D \times B_{1}\right)
$$

$\psi(f)=G$, where

$$
G(\theta, u)=\sum_{i=1}^{\infty} a(i) \theta(i) f(i), u(i)
$$

for all $f \in 2^{p}, q_{\langle E\rangle}$ and $\theta \in \pi D$, and $u \in B_{1}$. It follows that

$$
\begin{aligned}
\|G\|_{\infty} & =\sup _{\theta, u}|G(\theta, u)|=\sup _{\theta, u}\left|\sum_{i=1}^{\infty} a(i) \theta(i)\langle f(i), u(i)\rangle\right| \\
& =\sup _{u} \sum_{i=1}^{\infty}|a(i)\langle f(i), u(i)\rangle| .
\end{aligned}
$$

Hence $|F(f)| \leq\|\psi(f)\|$. This implies that ker $\psi \subseteq$ ker $F$.

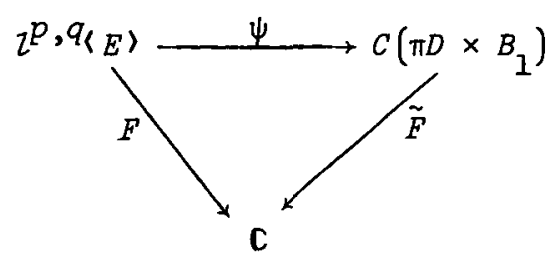

This implies that there exists an $\tilde{F}: C\left(\pi D \times B_{1}\right) \rightarrow \mathbb{C}$ such that $\tilde{F} \circ \psi=F$. The Riesz representation theorem implies that there exists a regular Borel measure $\mu$ on $\pi D \times B_{1}$ such that 


$$
F(f)=\mu(\psi(f))=\iint_{\pi D \times B_{1}} \sum_{i=1}^{\infty} a(i) \theta(i)\langle f(i), u(i)\rangle d \mu(\theta, u) .
$$

Let $f_{n}$ denote the function $f_{n}: N \rightarrow E$,

$$
f_{n}(i)= \begin{cases}f(i), & i=n, \\ 0, & i \neq n .\end{cases}
$$

Then

$$
F\left(f_{1}\right)=a(1) \iint_{\pi D \times B_{1}} \theta(1)(f(1), u(1)) d v(\theta, u) .
$$

But $F\left(f_{1}\right)=\left(f(1), x_{1}^{*}\right)$. It follows that

$$
x_{1}^{*}=a(1) \cdot \iint_{\pi D \times B_{1}} \theta(1) \cdot u(1) d v(\theta, u) \text {, }
$$

where the integral here is the Pettis integral, [4]. Set

$$
z_{1}^{*}=\iint_{\pi D \times B_{1}} \theta(1) u(1) d v(\theta, u)
$$

Hence $x_{1}^{*}=a(1) \cdot z_{1}^{*}$. Similarly $x_{i}^{*}=a(i) \cdot z_{i}^{*}, i=2,3, \ldots$. It remains to show that the function $g: N \rightarrow E^{*}$, defined by $g(i)=Z_{i}^{*}$, is an element of $Z^{q^{\prime}}\left(E^{*}\right)$. To see that, consider

$$
\begin{aligned}
|\langle g(i), x)| & \leq \iint_{\pi D \times B_{1}}|\theta(i)\langle u(i), x\rangle| d \mu(\theta, u) \quad(x \in E,\|X\| \leq 1) \\
& \leq \iint_{\pi D \times B_{1}}|\langle u(i), x\rangle| d \mu(\theta, u) \\
& \left.\leq \iiint_{\pi D \times B_{1}}|\langle u(i), x\rangle|^{q^{\prime}} d \mu(\theta, u)\right)^{1 / q^{\prime}} .
\end{aligned}
$$

Hence

$$
\sum_{i=1}^{\infty}|(g(i), x)|^{q^{\prime}} \leq \sum_{i=1}^{\infty} \iint_{\pi D \times B_{1}}|(u(i), x)|^{q^{\prime}} d \mu(\theta, u)
$$

The monotone convergence theorem implies that 


$$
\begin{aligned}
\sum_{i=1}^{\infty}|\langle g(i), x\rangle|^{q^{\prime}} & \leq \iint_{\pi D \times B} \sum_{i=1}^{\infty}|\langle u(i), x)|^{q^{\prime}} d \mu(\theta, u) \\
& \leq \sup _{u \in B_{1}} \sum_{i=1}^{\infty}|(u(i), x\rangle|^{q^{\prime}} \cdot|\mu|,
\end{aligned}
$$

where $|\mu|$ is the total variation of $\mu$. Thus $g \in 2^{q^{\prime}}\left(E^{*}\right)$. So $F=a \cdot g, a \in Z^{p^{\prime}}, g \in Z^{q^{\prime}}\left(E^{*}\right)$. This completes the proof of the theorem.

\section{Schur multipliers}

Let $p, q \geq 1$. A bounded function $\phi$ on $N \times N$ is called a schur multiplier of $\imath^{p} \hat{\otimes} l^{q}$ if $\phi \cdot \psi \in l^{p} \hat{\otimes} l^{q}$ for all $\psi \in Z^{p} \hat{\otimes} l^{q}$, where $\phi \cdot \psi$ denotes pointwise multiplication.

If $X$ and $Y$ are Banach spaces, then a bounded linear map $A: X \rightarrow Y$ is called p-suming operator if

$$
\sum_{i=1}^{n}\left\|A x_{i}\right\|^{p} \leq \zeta \cdot \sup _{x^{*}} \sum_{i=1}^{n}\left|\left(x_{i}, x^{*}\right\rangle\right|^{p},
$$

for all $x_{1}, \ldots, x_{n}$ in $X$ and some constant $\zeta$ independent of $n$. The supremum is taken over all elements $x^{*}$ in the unit ball of $X^{*},[10]$. Bennett [2] proved that a bounded function $\phi$ is a multiplier of $\imath^{p} \hat{\otimes} l^{q}$ if and only if $\phi \cdot u \otimes v: l^{p^{*}} \rightarrow l^{\infty}$ is $q^{*}$ summing operator for all $u \otimes v \in l^{\infty} \hat{\otimes} z^{p}$. For more about multipliers we refer to [2], [6], [7] and [3].

LEMMA 2.1. Let $A: \imath^{p} \rightarrow \eta^{\infty}$ be a bounded operator. If $A$ is $q$-swaming, then $A \in \tau^{q, q^{\prime}}\left(\tau^{p^{\prime}}\right)$.

Proof. Let $f: N \rightarrow 2^{p^{\prime}}$ be the function defined by $f(i)=A_{i}$, where $A_{i}(j)=A(i, j)$ (considering $A$ as an infinite matrix). If $g \in z^{q}\left(z^{p}\right)$, then 


$$
\begin{aligned}
\sum_{i=1}^{\infty}|(f(i), g(i)\rangle|^{q} & =\sum_{i=1}^{\infty}\left|\left\langle A_{i}, g(i)\right\rangle\right|^{q} \\
& =\sum_{i=1}^{\infty}\left|\sum_{j=1}^{\infty} A(i, j) g(i)(j)\right|^{q} \\
& \leq \sum_{i=1}^{\infty} \sup _{k}\left|\sum_{j=1}^{\infty} A(k, j) g(i)(j)\right|^{q} \\
& =\sum_{i=1}^{\infty}\|A(g(i))\|^{q} \\
& \leq \zeta \sup _{h} \sum_{i=1}^{\infty}|\langle g(i), h\rangle|^{q} \text { (by assumption), }
\end{aligned}
$$

where $h$ is the unit ball of $\imath^{p^{\prime}}$. Hence $f \in \tau^{q, q^{\prime}}\left\langle\tau^{p^{\prime}}\right\rangle$. Let $M\left(z^{p} \hat{\otimes} z^{q}\right)$ denote the space of all multipliers of $z^{p} \hat{\otimes} z^{q}$. Then:

THEOREM 2.2. Let $\phi$ be a bounded function on $N \times N$. Then $\phi \in M\left(z^{p} \hat{\otimes} z^{q}\right)$ if and only if $\phi \cdot 1 \otimes u \in z^{q^{\prime}, q\left(z^{p}\right\rangle}$, for all $u \in z^{p}$.

Proof. Let $\phi \in M\left(z^{p} \hat{\otimes} z^{q}\right)$. Then by Bennett's result [2], $\phi \cdot 1 \otimes u: l^{p^{\prime}} \rightarrow z^{\infty}$ is $q^{\prime}$-summing for all $u \in z^{p}$. Lemma 2.1 then implies that $\phi \cdot 1 \otimes u \in \imath^{\left.q^{\prime}, q_{\langle} \eta^{p}\right\rangle}$.

Conversely, let $\phi \cdot 1 \otimes u \in 2^{q^{\prime}, q}\left(z^{p}\right\rangle$ for all $u \in l^{p}$. It is enough to show that $\phi \in M\left(z^{q} \hat{\otimes} z^{p}\right)$. So let $u \otimes v \in z^{q} \hat{\otimes} z^{p}$, and $\psi \in \tau^{q^{\prime}} \imath^{p^{\prime}}$. Then

$$
\begin{aligned}
|\langle\phi \cdot u \otimes v, \psi\rangle| & =\left|\sum_{i, j=1}^{\infty} \phi(i, j) u(i) v(j) \psi(i, j)\right| \\
& =\left|\sum_{i=1}^{\infty} u(i)\left\langle\phi_{i} \cdot v, \Psi_{i}\right\rangle\right|,
\end{aligned}
$$

where $\phi_{i}(j)=\dot{\phi}(i, j)$ and $\psi_{i}(j)=\psi(i, j)$. Since $\psi \in \imath^{q^{\prime}} \imath^{p^{\prime}}$ it follows that $g: z^{+}+2^{p^{\prime}}$ defined by $g(i)=\psi_{i}$ is an element of $\imath^{q^{\prime}}\left(z^{p^{\prime}}\right),[3]$. Hence 


$$
\begin{aligned}
|\langle\phi \cdot u \otimes v, \psi\rangle| & \leq\|u\|_{q}\left(\sum_{i=1}^{\infty}\left|\left\langle\phi_{i} \cdot v, \psi_{i}\right)\right|^{q^{\prime}}\right)^{1 / q^{\prime}} \\
& \leq\|u\|_{q} \cdot\left\|\phi^{\prime} 1 \otimes v\right\|_{\sigma\left(q^{\prime}, q\right)^{\bullet}\|\psi\|_{\varepsilon\left(q^{\prime}\right)}} .
\end{aligned}
$$

This completes the proof of the theorem.

LEMMA 2.3. If $A: Z^{p^{\prime}} \rightarrow Z^{\infty}$ is $q^{\prime}$-summing, then $A \in M\left(\tau^{p} \hat{\otimes} l^{q}\right)$.

Proof. It is enough to show that $A I \otimes v: l^{P^{\prime}} \rightarrow l^{\infty}$ is $q^{\prime}$-summing operator for all $v \in Z^{p},[2]$. But

$$
\begin{aligned}
\sum_{i=1}^{\infty}\left\|(A \cdot 1 \otimes v) f_{i}\right\|_{\infty}^{q^{\prime}} & =\sum_{i=1}^{\infty}\left\|A\left(v \cdot f_{i}\right)\right\|_{\infty}^{q^{\prime}} \\
& \leq \zeta \sup _{h} \sum_{i=1}^{\infty}\left|\left(v \cdot f_{i}, h\right)\right|^{q^{\prime}} \\
& \leq \zeta \sup _{h} \sum_{i=1}^{\infty}\left|\left(f_{i}, v \cdot h\right)\right|^{q^{\prime}} \\
& \leq \zeta \sup _{k} \sum_{i=1}^{\infty}\left|\left(f_{i}, k\right)\right|^{q^{\prime}}
\end{aligned}
$$

where $h$ and $k$ are in the unit ball of $l^{p}$, and the lemma follows.

It follows from Lemmas 2.3 and 2.1 that the set of all $q^{\prime}$-summing maps from $l^{p^{\prime}}$ into $l^{\infty}$ is contained in $M\left(z^{p} \hat{\otimes} z^{q}\right) \cap l^{q}, q\left(z^{p}\right)$, where $n$ denotes the intersection of the two sets.

\section{References}

[1] Heikki Apiola, "Duality between spaces of $p$-summable sequences, $(p, q)$-summing operators and characterizations of nuclearity", Math. Ann. 219 (1976), 53-64.

[2] G. Bennett, "Schur multipliers", Duke Math. J. 44 (1977), 603-139.

[3] Joel S. Cohen, "Absolutely P-summing, P-nuclear operators and their conjugates", Math. Ann. 201 (1973), 177-200. 
[4] J. Diestel and J.J. UhI, Jr., Vector measures (Mathematical Surveys, 15. American Mathematical Society, Providence, Rhode Island, 1977).

[5] A. Grothendieck, "Sur certaines classes de suites dans les espaces de Banach et le théorèm de Dvoretzky-Rogers", Bol. Soc. Mat. São Paulo 8 (1953), 81-110 (1956).

[6] Roshdi Khalil, "Trace-class norm multipliers", Proc. Amer. Math. Soc. 79 (1980), 379-387.

[7] Roshdi Khalil, "On the algebra of multipliers", Canad. J. Math. (to appear).

[8] Roshdi Khalil, "Pointwise multipliers", J. Univ. Krwait Sci. (to appear).

[9] Gottfried Köthe, Topological vector spaces. I (translated by D.J.H. Garling. Die Grundlehren der mathematischen Wissenschaften,

159. Springer-Verlag, Berlin, Heidelberg, New York, 1969).

[10] J. Lindenstrauss and A. Pelczynski, "Absolutely summing operators in $L_{p}$-spaces and their applications", Studia Math. 29 (1968), 275-326.

[11] Albrecht Pietsch, Theorie der Operatorenideale (Zuscomenfassung) (Wissenschaftliche Beiträge der Friedrich-Schiller-Universität Jena. Friedrich-Schiller-Universität, Jena, 1972).

[12] H.L. Royden, Real analysis (Macmillan, New York; Collier-Macmillan, London; 1963).

Department of Mathematics,

University of Kuwait,

PO Box 5969,

Kuwait. 